\title{
OPTIMIZING A NONLINEAR COLLIMATION SYSTEM FOR FUTURE LINEAR COLLIDERS*
}

N. Merminga, J. Irwin, R. Helm, and R. D. Ruth

Stanford Linear Accelerator Center, Stanford University, Stanford, CA 94309 USA

\section{Abstract}

Experience indicates that beam collimation wis ${ }_{-j}$ an essential element of the next generation $e^{+} e^{-}$linear cc.lidcrs. A proposal for using nonlinear lenses to drive beam tails to large amplitudes was presented in a previous paper [1]. Here we study the optimization of such systems including effects of wakefields and optical aberrations. Protection and design of the scrapers in these systems are discussed.

\section{INTRODUCTION}

Experience with the SLC has indicated that backgrounds caused by transverse and energy tails of the beam distribution will be a fundamental problem of next generation linear colliders. Any collimation design must satisfy the following requirements:

1. It must provide an effective scraping despite the small (of the order of a micron) beam sizes. It should acrape particles with transverse positions greater than $5 \sigma$ in botlı planes as well as energy tails.

2. It must protect scrapers against mus-steered beams which may hit them and possibly damage them. There are two problems associated with a train of ten bunches of $10^{10}$ electrons per bunch at $250 \mathrm{GeV}$ hitting a scraper [2]. The first problem occurs at the surface of the scraper whicl may' melt because of energy deposited in a small area. More quantitatively we are interested in the largest spot size to cause failure of the scraper surface. If tailure is defined as the melting temperature of the material, then for $T i$, which is one of the best candidates according to SLC experience, the area to cause failure is [2]

$$
\sigma_{x} \sigma_{y} \simeq 900 \mu \mathrm{mi}^{2}
$$

The second problen occurs within the body of the scraper where the energy deposition from the sliower peaks, typically at several radiation lengths (RL) $(\simeq 8 \mathrm{RL}$ for Ti).

3. It must leep scraper-induced wakefield kicks on the beam below a tolerable level. Ir the beam does not pass exactly through the middle of the scrapers, it gets transverse deflections due to geometric and sesistive wall wakefields. If these kicks are comparable to the angular divergence of the beam, the emittance will increase.

An expression for the kick of the beam due to geometric wakefields which includes the effect of both edges of a scraper has been derived analytically and verified nuinerically [3] under the assumptions that the scraper gap is small compared to the scraper length, and the bunch length $\sigma_{z}$ is greater than or equal to the scraper gap. It is also assumed that the transverse deflection of a particle is produced by the dipole wakefield only and hence it is proportional to $\Delta\langle y\rangle / g$ where $\Delta\langle y\rangle$ is the beam offset from the middle of the scrapers and $2 g$ is the scraper gap. This expression is given by

$$
\Delta y^{\prime}=\theta_{\max }(\Delta\langle y\rangle / g)
$$

where

$$
\theta_{\max }=\sqrt{B / \pi}\left[\left(N r_{e}\right) /\left(\gamma \sigma_{z}\right)\right] \text {. }
$$

$N$ is the beam intensity and $\gamma=E / m c^{2}$. Using lypiral parameters for the Next Linear Collider (NLC), namely $N=1 \times 10^{10}$ particles per bunch, $\sigma_{z}=75 \mu \mathrm{m}, E=$ $250 \mathrm{GeV}$, we arrive at.

$$
\Delta y^{\prime}=1.2 \times 10^{-6}(\Delta(y) / g) \mathrm{rad}
$$

T Work supported by Department of Energy contract DE-AC03-765F00515.
To reduce the effect of the geometric wakefield kick, one can taper the scrapers with a taper angle $\theta_{\text {tap }},\left(\theta_{\text {tap }}=\right.$ $\pi / 2$ for a step scraper.) For small taper angles ( $\theta_{\text {esp }} \leq$ $100 \mathrm{mrad})$ the dependence on the taper angle is linear $[4,5]$,

$$
\Delta y^{\prime}=2 \theta_{\text {tap }} \theta_{\max }(\Delta(y) / g)
$$

The kick due to the resistive wall wakefield is proportional to $\Delta\langle y\rangle / g^{3}$. Specifically it is given by

$$
\Delta y^{\prime}=C_{\max }\left(\Delta(y) / g^{\mathfrak{3}}\right) L_{\mathrm{scr}}
$$

where $L_{\mathrm{scr}}$ is the scraper length,

$$
C_{\max }=(4 / \pi)\left(N r_{e} / \gamma\right)[(c / \sigma) / 1]^{1 / 2} f(s),
$$

$\sigma$ is the conductivity of the material, $l=2 \sigma_{1}$, and $f(s)$ is a function of the longitudinal coordinate within the bunch, varying between 0 and 1 . For typical NLC parameters and for a scraper made of $\mathrm{Ti}$,

$$
\Delta y^{\prime}=0.85 \times 10^{-13}\left(\Delta(y) / g^{3}\right) L_{\text {ser }} \text { l.ad }
$$

The function $f(s)$ has been approximated by $1 / 2$ to account for the head to tail variation of the wakefield. For small gaps this is the dominant wakefield effect.

On the basis of the above issues we have demonstrated [6] that mechanical collimation is precluded for the vertical degree of freedom as a workable collimation technique for the NLC. In the following section we present the nonlinear collimation scheme as a possible alternative. We first write the conditions that must be satisfied. These conditions deternine a set of lattice parameters for the collimation systems. Then we present a possible lattice design, calculate its tolerances and discuss our ideas on energy collimation.

II. NONLINEAR COLLIMATION IN THE NLC: SCHEME WITH SKEW SEXTUPOLE PAIRS

The basic idea $[1,6]$ is to use several nonlinear lenses to drive the tails of the beam distribution to large amplitudes where they can be scraped mechanically. In the NLC collimation is proposed to be done mechanically in the horizontal plane and nonlinearly in the vertical plane (scheme with skew sextupole pairs). The horizontal scrapers will be placed at high horizontal beta function points, interleaved with the vertical scrapers. Energy scraping takes place right after transverse scraping. A schematic representation of the collimation section of the NLC is shown in Fig. 1

The collimation design must satisfy all of the following conditions.

a. It must scrape transverse tails beyond $5 \sigma$ in botli planes.

b. It must scrape energy tails.

c. Resistive wall wakes at both horizontal and vertical scrapers must be controlled.

d. Geometric wakes at both horizontal and vertical scrapers must be controlled.

e. Geometric and resistive wall wakes at the sextupoles must be controlled.

f. Long sextupole aberrations must be controlled.

g. It must ensure protection of horizontal, vertical and energy scrapers.

h. Stability tolerances on sextupole and scraper offsets nust be acceptable.

i. The collimation systens must not create unacceptable optical aberrations.

Next we elaborate on each of the above conditions and thus arrive at the allowed design paraneters of the collimalion system. 


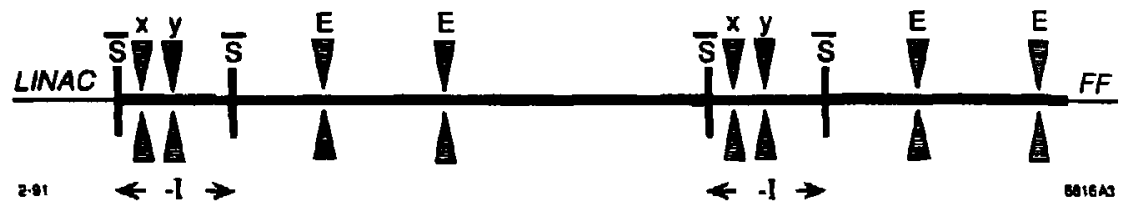

Figure 1. Schematic representation of the collimation systems in the NLC, located between the linac and fina focus (FF).

$S$ stands for skew sextupole; $x, y, E$ stand for horizontal, vertical and energy seraper respectively.

\section{Scraping in the vertical plane}

This condition implies that particles whose vertical coordinates are greater or equal to $5 \sigma_{y}$ at the sextupole must be mapped into vertical positions greater or equal to $g_{y}$ at the scraper,

$$
\Delta y_{\text {sec }}\left(\left|y_{\text {sext }}\right| \geq 5 \sigma_{y, \text { sext }}\right) \geq g_{y}
$$

A $5 \sigma$ particle at the skew sextupole will experience a kick

$$
\Delta y_{\text {sext }}^{\prime}=S\left(5 \sigma_{y}\right)^{2}
$$

where $S$ is the integrated sextupole strength. This kick will in turn give rise to an offset at the scraper

$$
\Delta y_{\text {ace }}=R_{12} \Delta y_{\text {sext }}^{\prime} \text {, }
$$

where $R$ is the transfer matrix between sextupole and scraper. Combining the above equations we arrive at the condition

$$
25 S \epsilon_{y} R_{12} \rho_{\text {cext }} \geq g_{y} \text {. }
$$

Resistive wall wakes at the vertical scrapers

As we showed earlier the resistive wall wakefield kick at the scraper is given by

$$
\Delta y_{\text {icr }}^{\prime}=C_{\max }\left(\Delta\left(y_{\text {ecr }}\right) / g_{y}^{3}\right) L_{\text {acr }}
$$

which becomes at the downstream sextupole

$$
\Delta y_{\text {next }}=R_{12} \Delta y_{\text {ser }}^{\prime}
$$

An offset through the skew sext upole gives rise to a normal quadrupole kick of magnitude

$$
\Delta y_{\text {bext }}^{\prime}=\left(2 S \Delta y_{\text {sext }}\right) y
$$

We require that the rms value of these kicks be less than $1 / 5 \sigma_{y}^{\prime}$ (to avoid unacceptable longitudinal jitter of the final focal point),

$$
\left(2 S \Delta y_{\text {next }}\right) y_{\text {rms }} \leq(1 / 5) \sigma_{y}^{\prime} \text {. }
$$

We wish to allow a $1 \sigma$ jitter of the incoming beam centroid, hence we take

$$
\Delta\left(y_{s c r}\right)=\sigma_{y, \text { acr }}
$$

in eq. (16), which combined with eqs. (13) and 14) gives

$$
2 S C_{\max } \epsilon_{y}^{1 / 2} R_{12}^{2} L_{\text {acr }} \beta_{\text {sext }}^{1 / 2} \leq(1 / 5) g_{y}^{3}
$$

Long sextupole aberrations

The polential for long-sextupole aberrations is given by

$$
V_{L S}=(1 / 12) S^{2} L_{\text {sext }} y^{4}
$$

assuming small horizontal beam size. Therefore the longsextupole kick is

and we require

$$
\Delta y^{\prime}=(1 / 3) S^{2} L y^{3}
$$

$$
\left(\Delta y^{\prime}\right)_{\mathrm{ms}} \leq(1 / 5) \sigma_{y}^{\prime} \text {. }
$$

This leads to the condition

$$
(5 \sqrt{15} / 3) S^{2} L_{\text {sext }} \varepsilon_{y} \beta_{y, \text { sext }}^{2} \leq 1 \text {. }
$$

For the two sextupoles of the $-l$ transformation, the above equation determines the maximum allowed vertical $\beta$-function, $\beta_{y, \text { ext }} \leq 23,000 \mathrm{~m}$. In deriving this, we have assumed a pole-tip field of 1 Tesla, pole-tip radius of $1 \mathrm{~mm}$, and sextupole length of $10 \mathrm{~cm}$.

Equations (12), (18), and the long sextupole aberration limit determine the parameter space for the vertical plane, once the values of $R_{12}$ and $L_{\text {scr }}$ are specified. The scraper length was chosen to be equal to $3 \mathrm{RL}$ of $\mathrm{Ti}$, namely
$11.3 \mathrm{~cm}$. To arrive at this value we used the code EGS [9] to calculate the number of electrons that make it through the $3 \mathrm{KL}$ of $\mathrm{Ti}_{1}$ with energies between 245 and $250 \mathrm{GeV}$. We found that one out of $10^{12}$ electrons belongs to this energy bin. Although more accurate EGS calculations should be performed, these preliminary results indicate that $3 \mathrm{RL}$ of Ti change the beam energy sufficiently in order for subsequent energy scraping to collimate the beam.

The value of $R_{12}$ is directly related to the total length of the systen and lience it should be kept minimum. For $R_{12}=50 \mathrm{~m}$ (which corresponds to a length between sextupole and scraper of about $30 \mathrm{~m}$ ), and an $11.3 \mathrm{~cm}$ long scraper we plotted the above equations in Fig. 2. The region $A$ enclosed by the three curves corresponds to the allowed space. Now we can choose the parameters of the collimation design in the vertical plane: $\beta_{\text {y.sext }}=6,000 \mathrm{~m}$ and $g_{y}=90 \mu \mathrm{m}$. For this choice of parameters the geometric wakefield condition at the vertical scrapers is satisfied. We need however to taper the beam pipe at the sextupoles by a 15 mrad angle. The resistive wall wakefield condition is satisfied at the sextupoles.

\section{Horizontal consideratjons}

A $n$ important consideration that determines the x-plane parameters is the $x-y$ coupling at the sextupoles. To minimize coupling effects we must ensure that at the sextupoles,

$$
S y^{2} \gg S x^{2}
$$

which establishes a condition on $\beta_{x}$ at the sextupoles,

$$
\beta_{x, \text { next }} \ll \beta_{y, \text { eext }}\left(\epsilon_{x} / \epsilon_{y}\right)
$$

2.: our case, $\beta_{ \pm}$sext $\ll 60 \mathrm{~m}$. If we place the horizontal scrapers at a relatively high $\beta_{y}$ point, i.e., at $\beta_{y}=600 \mathrm{~m}$. then in order to ensure scraper protection, $\beta_{x}$ at this point must be greater than $1400 \mathrm{~m}$. In fact we chose $\beta_{x, s c r}=$ $2,000 \mathrm{~m}$, which implies a scraper gap of $700 \mu \mathrm{m}$ for $5 \sigma_{x}$ scraping. Once the $\beta$-function at the scraper is fixed, the $\beta$-function at the sextupoles follows, $\beta_{r \text { sext }}=0.1 \mathrm{~m}$.

We now address the question of geometric and resistive wall wakefields at the horizontal scrapers. Again here both wakefield kicks must be below $1 / 5 \sigma_{x}^{\prime}$. These conditions are simultaneously satisfied if the horizontal scrapers, assumed 10-cm long, are tapered by an angle of $30 \mathrm{mrad}$ Each tapered section of the scrapers is then $15-\mathrm{cm}$ long. Finally, the horizontal geometric wakefield condition at the sextupoles is satisfied.

\section{Lattice-energy collimation}

A lattice design which satisfies the above specifications is presented in Fig. 3. It starts with a -1 transformation where horizontal and vertical scraping of the first phase space direction takes place. This is followed by a $2 \pi$ sertion dedicated to energy collimation. Next there is a $3 \pi / 2$ in the horizontal plane and $\pi / 2$ in the vertical plane transformer section. A prise advance of $\pi / 2$ in both planes would have been possible at the expense of considerable increase in length. The last section of the line is identiral to the first one. It is used to scrape the second pliase space direction and energy again. The total length of the systemn is about $500 \mathrm{~m}$. 


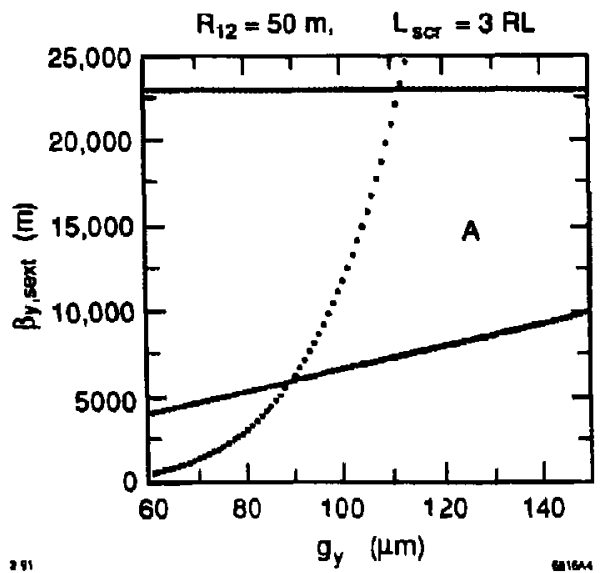

Figure 2. Parameter space for nonlinear collimation with sextupoles.

Energy collimation is done by transforming off-energy particles to large amplitude ones through the introduction of horizontal dispersion. There are two scrapers in each energy scraping section placed at high dispersion points. The horizontal and vertical $\beta$ functions at these locations are the same as the ones at the horizontal scrapers. Both energy scrapers consist of a thin $(\simeq 3 \mathrm{RL}$ ) and a thich part $(\simeq 20 \mathrm{RL})$. The thin part will be responsible for the primary beam energy collimation. By making it thin we bypass protection problems that occur within the body of the scl aper. The role of the thick part will be to absorb the debris from both horizontal and energy collimation that has occurred upstream.

Furthermore, each of the two energy collimation sections includes a normal sextupole pair forming a $-I$ transformation. Their function is to correct the horizontal chromaticity. To correct the vertical chromaticity a small amount of vertical dispersion has been added to the lattice at the skew sextupoles. Simulations show that this entire lattice demonstrates an excellent behavior with respect to chromatic and chromo-geometric aberrations in both transverse planes.

\section{Stability tolerance on scraper offset}

In deriving some of the above conditions, we have assumed that the offset through the middle of the scraper is of the order of the beam size. Since the beam size at the vertical scrapers is $0.20 \mu \mathrm{m}$, the stability tolerance on the scraper offset is also $0.20 \mu \mathrm{m}$.

From eq. (8) one can estimate an absolute steering tolerance by requiring that

$$
\left(\Delta y^{\prime}\right)_{\text {rw }} \leq(1 / 5) \sigma_{y}^{\prime}, \text { aer }
$$

and solving for $\Delta y$. It turns out that this tolerance is $7.4 \mu \mathrm{m}$.

\section{Stability tolerance on sextupole offsets}

In order to get some insight into the question of tolerances we derive a general result for the tolerance on the sextupole oftset. If we combine the scraping condition eq. (12) with the requirement that the quadrupole-like kick due to the sextupole offset $y_{0, \text { sert }}$ must satisfy eq. (16), we arrive at

$$
y_{0, \text { sext }} \leq(5 / 2)\left[\left(R_{12} \epsilon_{y}\right) / g_{y}\right] \text {. }
$$

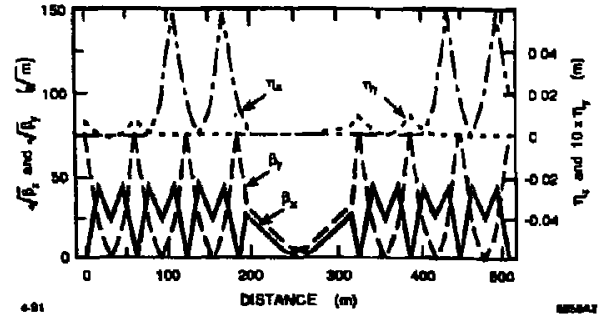

Figure 3. Optics design for the collimation systems in the NLC.

Notice that the only parameters that can affect this offset tolerance are effectively the length of the system (via $R_{12}$ ) and the scraper gap. For our choice of parameters this tolerance is $0.14 \mu \mathrm{m}$.

\section{Protection of scrapers}

As we mentioned in the Introduction there are two prob. lems associated with a train of bunches hitting the scrapers: the first occurs at the surface of the scraper while the second occurs in the body of the scraper. The surface of the scrapers is protected by design. More preeisely, at the horizontal scrapers the area occupied by one $\sigma$ of the beam is $1100 \mu \mathrm{m}^{2}$, beyond the $900 \mu \mathrm{m}^{2}$ limit quoted earlier.

At the vertical scraper, on the other hand, one can calculate the scraper area on which $1 \sigma_{x} \times 1 \sigma_{y}$ particies at the sextupoles are mapped, if the bearn is mis-steered by an amount greater than $5 \sigma$. It turns out to be $4,400 \mu \mathrm{m}^{2}$, far beyond the $900 \mu \mathrm{m}^{2}$ limit.

The problem of the body of the scrapers is solved by making the scrapers short, $3 \mathrm{RL}$ of the material.

\section{CONCLUSIONS}

We presented a possible collimation scheme for a $\mathrm{TeV}$ linear collider which employs mechanical collimation for the horizontal plane and nonlimear collimation (sclieme with skew sextupole pairs) for the vertical. This design succeeds in satisfying all of the requirements imposed on collimation systems, including effective collimation of transverse and energy tails, control of wakefield effects, protection of scrapers, and conirol of geometric and chromatic aberrations. The stability tolerances at the scrapers and sextupoles are similar to those occurring in the NLC Final Focus system; given the precision of the beam position inonitors envisioned for an NLC, these tolerances should not rule out nonlinear collimation as a candidate for beam scraping in a future linear collider.

\section{Acknowledgements}

The authors would like to thank K. Yokoya of KEK for pointing out the importance of the resistive wall wakefield effect, and R. Nelson of SLAC for his prompt response to our request for EGS calculations.

\section{REFERENCES}

(1) N. Merminga and R. Ruth, Proc. 1990 EPAC, p. 1738.

[2] D. Walz, private communication.

[3] K. Bane and P. Morton, SLAC-PUB-3983, 1980.

[4] K. L. F. Bane, privale communication.

[5] K. Yokoya, CERN SL/90-88(AP), 1990.

[6] N. Merminga and J. Irwin et al., SLAC-PUB-5436. 1991

[7] J. Jrwin, SLAC-PUB-5315, 1990.

[8] K. Oide, SLAC-PUB-4953, 1989.

[9] W. R. Nelson et al, SLAC-2.65, 1985. 


\section{DISCLAIMER}

This report was prepared as an uccount of work sponsored by an agency of the United States Government. Neither the United States Government nor any ageacy thereof, nor any of their employees, makes any warranty, express or implied, or assumes any legal liability of responsibility for the accuracy, completeness, or usefulness of any information, apparatus, product, or process disclosed, or represents that its use would not infringe privately owned rights. Reference herein to any specific commercial product, process, or service by trade name, trademart, manulacturer, of otherwise does nol necessarily constitute or imply its endorsement, recommendation, of favoring by the United States Government or any agency thereof. The views and opinions of anthors expressed hercin do not necessarily state or reflect those of the United States Government or any agency thereof. 